\title{
Treating Bulimia Nervosa by Dietary Modifications
}

\author{
Niyati Sheokand, Ved Mahla and Priya Arora \\ Department of Psychiatry Shree Guru Gobind Singh Tricentary \\ University, Gurugram, Haryana, India \\ Corresponding author email: niyatisheokand@gmail.com
}

\begin{abstract}
Bulimia Nervosa (BN) is a complicated eating ailment involves starving and consuming a lot of food. BNis a psychiatric eating ailment. Patient suffering from Bulimia Nervosa plans to follow a strict diet, under starving condition person starts binge eating. Binge eating makes the person feel guilty about eating. The feeling of guilt increases to an extent, results in person to self-induce vomiting and follow other ways to get rid of calories consumed by binge eating. As a psychiatric and eating disorder the treatment is very difficult. Difficulty in treating patient may be because of trust issue, depression, comorbidity, personality disorder and many other things. Treatment of Bulimia Nervosa by providing medications involving antidepressant, is solely not effective. A combination of healthy diet containing food rich in nutrients required to reverse the condition is suggested although more clinical studies are required to be conducted.
\end{abstract}

KEY WORDS: BINGE, BULIMIA NERVOSA,PURGE, SEROTONIN.

\section{INTRODUCTION}

Bulimia Nervosa/BN is a complicated eating ailmentwhich involves eating a lot of food, and later person works hard to get rid from the food consumed. Person suffering from Bulimia Nervosa generally eat a lot of food. Quantity of food consumed by $\mathrm{BN}$ patient at one time is much more than the quantity of food consumed by other person of same age group and gender. Bulimia Nervosa patients generally lose control while eating and stops when the food is consumed. Consuming a lot of food puts the patient in guilt and then the patient puts efforts to get rid of food they have consumed. In order to get rid of the calories, person has consumed through diet, patient induce vomit. Other ways to get rid of calories consumed through diet is using laxatives, enemas, diuretics and other medications also.

Patients also choose to fast for few days after a bad binge. Some patients do excessive exercise to burn the calories

Biosc Biotech Res Comm P-ISSN: 0974-6455 E-ISSN: 2321-4007
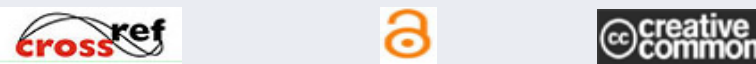

Identifiers and Pagination

Article Information

Year: 2021 Vol: 14 No (6) Special Issue

Received: $15^{\text {th }}$ May 2021

Pages: $362-365$ Accepted after revision: $19^{\text {th }}$ July 2021

This is an open access article under Creative

Commons License Attribn 4.0 Intl (CC-BY).

DOI: $h t t p: / / d x . d o i . o r g / 10.21786 / b b r c / 14.7 .76$ consumed. Bulimia Nervosa patients are not easy to recognise, as the weight of $\mathrm{BN}$ patients generally remains in normal body weight range. Public eating behaviour of $\mathrm{BN}$ patients is also very normal, they either eat less or don't eat in public gathering. Binge eating is always done when the patient is alone because of the fact that they are very conscious about their public image. (A. S. Kaplan (1999), A. Carmosino). Figure 1 shows binge and purge cycle of Bulimia Nervosa patient.

The severity of psychiatric and medical condition is a result of social pressure of looking thin and increasing role of media in showing thinness as superior quality. Commercial advertisements shows models with lean body only, is also a reason for Anorexia Nervosa in teenagers. People already suffering from genetic disorders are more prone to get affected. Social pressure along with genetic disorders makes a person highly susceptible for AN (M.B et. al. (1997).

Media has an important role on people's mind-set as many women, teenagers starve themselves to look lean. Commercial advertisements also show perfect image of a girl or boy with lean body. The pressure of looking lean and fitting in the ideal figure created by media is also responsible for severity in patients and high mortality rate. According to Becker et al, study conducted on eating behaviour of Fijian adolescent girls, 17 years age, from 1995 to1998 and it is 
observed that high television exposure is the reason for self-induced vomiting and dieting behaviour. (A. E. Becker et. al. (2002))

1. Eating disorder statistics worldwide: Anorexia is a highly scary ailment related to brain. According to study, anorexia patients are fifty six times more prone to take their life than patients having any other ailmentand not any eating disease. Prevalence of ailment related to eating ailments raised from $3.4 \%$ to $7.8 \%$ from 2000 to 2018 globally. 70 million people internationally live with eating ailments. The highest prevalence of eating ailment is observed to be highest in Japan in Asia. After japan Singapore, South Korea, Taiwan, Hong Kong showed high rate of eating ailment. In Europe Austria is observed to be showing highest rate of eating ailment in Europe at 1.55 percent in year 2012

2. Eating disorder statistics by sex: Eating disorders in young women is $3.8 \%$ and $1.5 \%$ in men, the U.S. as of $2001-$ 2004. 25\% patients are male. Men are observed to be at a greater risk of dying due to eating ailment due to the fact of delay in diagnosis in comparison to women. The delay in diagnosis observed in men is the result of the assumption that men usually do not suffer from eating ailments.

3. Eating disorder statistics by age: A worldwide statistic observed that women more than 50 years of age are 13 percent more prone to suffer from eating ailment. The average age of the start of the eating ailment was observed to be twenty one years for ailment related to consuming excess food and eighteen years for anorexia and bulimia nervosa. According to data collected the spreading of eating ailment in United States of America was observed to be 2.7 percent in case of adolescents in year 2001 to 2004 and out of this populating in the age group of seventeen to eighteen years showed maximum prevalence rate as three percentage( $M$. Anis Rehamn et. al.).

\section{Physical Complications:}

4.1. Erosion of Tooth Enamel: Induced vomiting results in repeated acid attack on teeth as a result erosion of tooth enamel is observed.Dental cavities: In order to avoid food intake, patients generally spit and chew which results in dental cavity.

4.2. Tooth Sensitivity: Enamel of teeth mainly works to protect teeth from decay, forced vomiting causes acidic attack on teeth results in sensation towards cold and hot eatables.

4.3. Soreness And Inflammation In The Saliva Producing Exocrine Glands: Swelling in salivary glands as a result of repeated vomiting and nutritional deficiency is observed.

4.4. Stomach Ulcers: Repeated binging and purging results in ulcers in stomach.

4.5. Ruptures of the Stomach and Oesophagus: Starving for a long period of time and then binging followed by purging, behaviour against the natural digestion process affects stomach heavily. Induced vomit results in rupture of oesophagus also.

4.6. Disruption in the Normal Bowel Release Function: Nutritional deficiency along with binge eating and immediate purging disrupts normal bowel function

4.7. Dehydration: Use of laxatives, diuretics, binging and purging results in electrolyte imbalance in the body.

4.8. Irregular Heartbeat: Heartbeat irregularity is related to dehydration and lack of electrolytes and nutritional deficiency in body.

4.9. Heart Attack (in severe cases): Dehydration, nutritional deficiency, electrolyte imbalance and purging all activities affect heart activity and ultimately cause heart attack.Higher risk for suicidal behaviour(Smitha Bhandari et. al.).

Review Of Literature: Shapiro et al.conducted trials to study the effectiveness of a medicine on Bulimia Nervosa, few patients showed reduced symptom 1 whereas few patients showed reduced symptom 2 . The current study does not suggest a medicine with no side effects for Bulimia Nervosa, applicable for all age group of affected population (J.R. Shapiro et. al. (2007).Rosen et al.conducted study and analysed the symptoms of Bulimia Nervosa and suggested that a specific amount of food when consumed does not result in vomit after binge eating. The current study does not give an idea about the link between food and brain chemical imbalance (J. C. Rosem et. al. (1982). Fairburn et al.conducted a study and find the cognitive behavioural therapy for bulimia nervosa. The current study does not provide link between food and brain chemical balance and a no side effect treatment for bulimia nervosa (C. G. Fairburn et. al. (1993)).

Figure 2: Chemical structure of Serotonin, 5-hydroxytryptamine (5-HT) monoamine neurotransmitter also known as happiness hormone(M. Aliouche et. al.).

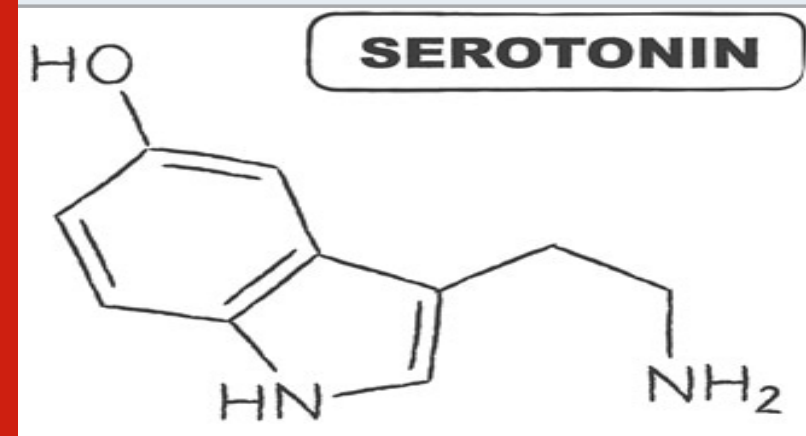

5-hydroxytryptamine (5-HT)

METHODOLOGY

Lean body is considered superior and fat body as inferior, a mind-set created by media and society is creating a mental pressure on people to maintain lean body or to look lean. Population having genetic history of Bulimia nervosa are 
more prone to suffer from the disease. Many studies suggests an important role of female hormone in Bulimia Nervosa. Recent studies shows the link of stress as an important factor in Bulimia Nervosa in females. Early studies connect the symptom of binge eating as a result of Bulimia Nervosa in females during mensuration. Studies also show that not every women binge eat during mensuration, although every women go through the same hormonal changes during mensuration cycle. Studies conducted by Natasha fowler et al. link Bulimia Nervosa with stress.(B. A. Hildebrandt (2015), Natasha Fowler).

1. Serotonin: Serotonin neurotransmitter and hormone supports in regulating sleep along with mood, hunger, digestion function, learning skills, and memory in human beings (Figure 2). Serotonin is the in-charge for feelings of calmness and happiness. Excessive secretion of serotonin results in anxiety, whereas very less quantity of serotonin secretion result in feeling of sadness and depression like symptoms. The role of serotonin in $\mathrm{BN}$ patients, there are evidences that suggests that disturbance in serotonin functioning in brain is responsible for the alteration of diet, impulse control, and mood, in anorexia and bulimia. High level of serotonin in body may eventually result increased satiety, thus the person feels full as a result person starve. As a result of weight loss serotonin level decreases inside the brain.

Weight loss results in short term alleviation from negative thoughts and emotional dis balance which strengthens the symptoms. (Timothy J. Legg et. al. (2019). K. A. Smith and P. J. Cowen, studies suggests that, a medium dieting for 21 days reduced plasma levels of Tryptophan. Tryptophan, an amino acid responsible for production of serotonin. In recovered subjects, it was found that people already suffered from bulimia nervosa depletionin Tryptophan amino acid resulted in a short term re-occurrence of depressionlike symptoms. Concerns regarding weight and shape and danger of un-controlled eating were also analysed. (P.J. Cowen et. al. (2000).

Figure 3: Synthesis of serotonin(How is serotonin synthesised in the body? et. al.).

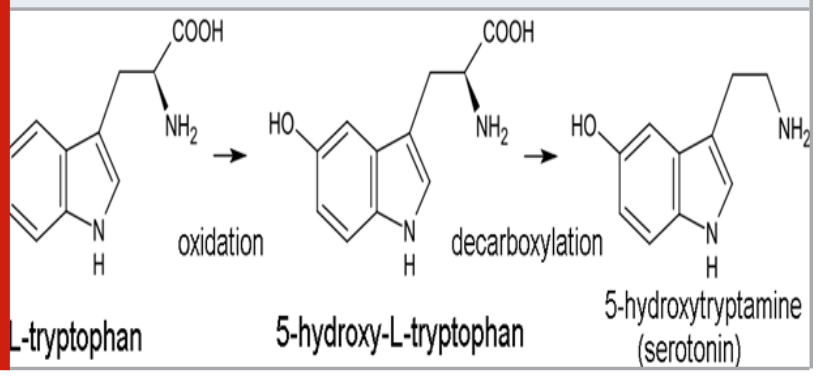

2. Serotonin synthesis: Serotonin synthesis inside the body takes place from tryptophan. Tryptophan, as mentioned in Figure 3 is an essential amino acid (EAA). EAA can only be provided to the body when consumed through diet. Deficiency of tryptophan in diet results in less production of serotonin in the body. Serotonin controls sleep, mood appetite, learning ability, digestion, and memory. As a result of low levels of serotonin in the body, every activity of human body controlled by serotonin gets affected. (Y. 13 Jo et. al.). Use of antidepressant: Medicines prescribed for the treatment of Bulimia Nervosa include antidepressants. Antidepressants instantly boost mood and also give some side effects which a patient cannot tolerate. In severe cases, it difficult for a patient to reverse the condition of Bulimia. Prescribing high dose of antidepressant to adolescent patients is also not possible. Severe side effects of antidepressants and non-tolerance led to find a solution for Bulimia Nervosa patients of all age group. Figure 4 gives a brief idea about the mechanism of action of antidepressants.

Figure 4: Mechanism of action of Selective Serotonin Reuptake Inhibitors(SSRIs) also known as antidepressant(N. Kaciroti et. al.)

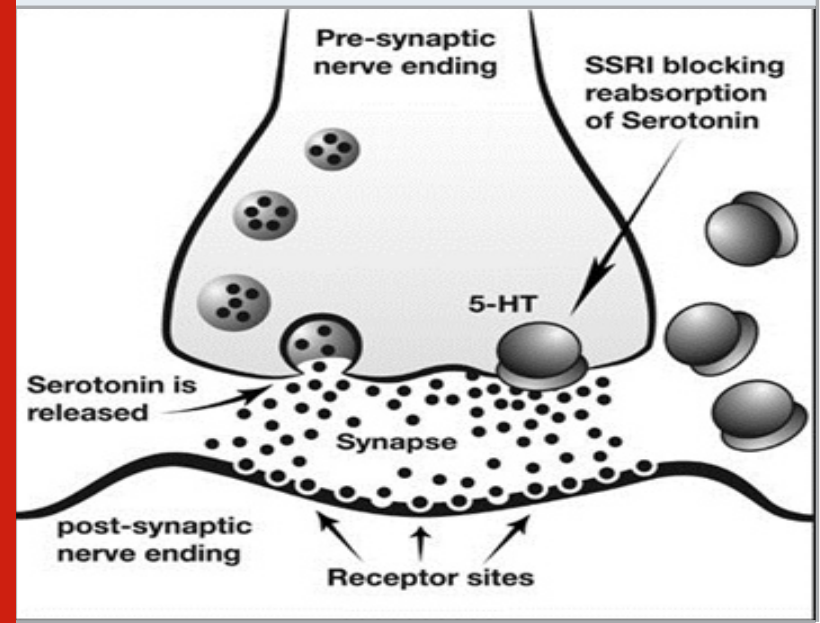

Treatment of Bulimia Nervosa: By giving a healthy balanced diet to the patient along with psychotherapy and counseling. The psychotherapy and counseling sessions also include convincing patient to eat a balanced diet. Figure 5 gives an idea about essential and non-essential amino acids. Amino acids which are not produced in the body of a human being and are required to be consumed through diet are called essential amino acids. Any prolong deficiency of essential amino acids in diet results in hormonal imbalance. As depicted in Figure 5, essential amino acids are linked with food items shows that the source of essential amino acids are food items and can only be consumed by balanced diet. Amino acids which are synthesised inside the body are non-essential amino acids, although these are not nonessential as the name suggests, the name only shows that non-essential amino acids are also synthesised inside the body. The only source of essential amino acids (EAA) is diet but non-essential amino acids can also be synthesised inside the human body.

A prolong disturbance in the brain chemistry results in person to behave differently without knowing the reason behind it. The solution to normalize the brain chemical imbalance in Bulimia Nervosa patients is psychotherapy along with counselling and convincing the patient to eat a balanced diet. Although, in severe cases patients do not accept eating a balanced diet and body also don't adapt digesting food provided in a given quantity regularly. To 
overcome this, a small portion of protein per gram of body weight is provided for a week time and then it is monitored. After 7 to 10 days patient's diet plan is changed with slight increased protein content, again this revised diet chart is monitored for 7 days.

Figure 5 : Representation of the list of essential amino acids and non-essential amino acids(S. G. Christensen et. al.)

\begin{tabular}{|l|l||}
\hline Non-Essential Amino acid \\
Alanine \\
Arginine \\
Asparagine \\
Cysteine \\
Glutamic \\
Glycine \\
Ornithine \\
Proline \\
Serine \\
Tyrosine
\end{tabular}

Now, the patient after eating protein rich food for over 15 to 20 days is now in a condition to gain weight. Even if the patient did not gain weight, after eating protein rich diet for around 20 days behavioural symptoms might be reduced as a result of presence of tyrosine and tryptophan in the diet. Essential amino acids responsible for balance of dopamine and serotonin in body when consumed through diet results in chemical balance in brain also. As per Indian Council of Medical Research (ICMR), (C. Gopalan et. al. (1987)) the recommended dietary allowance (RDA) of protein for Indians is:

Girls (16-17 years) - 1.065 grams of protein per $\mathrm{kg}$ of person's body weight.

Boys (16 -17 years) - 1.11 grams of protein per $\mathrm{kg}$ of person's body weight

Men/ women - 1 gram of protein per kg of person's body weight.

In United States, recommended dietary allowance (RDA) of protein is Men/Women - 0.8 gram per $\mathrm{kg}$ of person's body weight (M.D.-W et. al.)

A good source of animal protein is suggested - egg, fish, milk and its products to get fast and better results. A mixed diet of plant protein - combination of cereals, pulses and green vegetables is suggested.

Initially, patient should be allowed a diet plan containing 0.65 to 0.7 gram of protein per $\mathrm{kg}$ of body weight for 7 to 10 days.

After monitoring the above plan, it is suggested to increase the protein diet to 0.85 to 0.9 gram per $\mathrm{kg}$ of body weight for 7 to 10 days.

Now, the patient is provided with 1 to $1.065 / 1.1$ gram of protein per kg of person's body weight.

\section{CONCLUSION}

Restriction of food intake to look lean in Bulimia Nervosa patients results in chemical imbalance in brain. Chemicals responsible for major body functions are involved in Bulimia Nervosa. To maintain the brain chemical balance a good protein rich diet is suggested. Amino acids in body, generally obtained by diet are restricted by patient as a result of less food intake and induced vomiting after binging. A well monitored and balanced diet with good protein quantity is suggested. The conclusion is drawn from the above studies that lack of food creates chemical imbalance in human brain and further creates complications in the patient. A protein rich diet can reverse the condition by providing nutrition to the body.

\section{REFERENCES}

Cowen, P.J. and Smith, K.A., 1999. Serotonin, dieting, and bulimia nervosa. Tryptophan, Serotonin, and Melatonin, pp.101-104.

Fairburn, C.G., Marcus, M.D. and Wilson, G.T., 1993. Cognitive-behavioral therapy for binge eating and bulimia nervosa: a comprehensive treatment manual.

Fowler, N., Vo, P.T., Sisk, C.L. and Klump, K.L., 2019.

Stress as a potential moderator of ovarian hormone influences on binge eating in women. F1000Research, 8. Gopalan, C., Rama Sastri, B.V. and Balasubramanian, S.C., 1971. Nutritive value of Indian foods.

Hildebrandt, B.A., Racine, S.E., Keel, P.K., Burt, S.A., Neale, M., Boker, S., Sisk, C.L. and Klump, K.L., 2015. The effects of ovarian hormones and emotional eating on changes in weight preoccupation across the menstrual cycle. International Journal of Eating Disorders, 48(5), pp.477-486.

Kaplan, A.S., 1999. Garfinkel, PE. Difficulties in treating patients with eating disorders: A review of patient and clinician variables. Can J Psychiatry, 44, pp.665-70.

Lattimore, K.A., Donn, S.M., Kaciroti, N., Kemper, A.R., Neal, C.R. and Vazquez, D.M., 2005. Selective serotonin reuptake inhibitor (SSRI) use during pregnancy and effects on the fetus and newborn: a meta-analysis. Journal of Perinatology, 25(9), pp.595-604.

Rosen, J.C. and Leitenberg, H., 1982. Bulimia nervosa: Treatment with exposure and response prevention. Behavior Therapy, 13(1), pp.117-124.

Shapiro, J.R., Berkman, N.D., Brownley, K.A., Sedway, J.A., Lohr, K.N. and Bulik, C.M., 2007. Bulimia nervosa treatment: a systematic review of randomized controlled trials. International Journal of Eating Disorders, 40(4), pp.321-336.

Striegel-Moore, R.H. and Bulik, C.M., 2007. Risk factors for eating disorders. American psychologist, 62(3), p.181. 